\title{
Development of a hardware-in- loop simulation platform for NPP main control systems
}

\author{
Pengfei Liư ${ }^{1, a}$, Chunyu $\mathrm{Bi}^{1}$, Yang $\mathrm{Yu}^{1}$, Meng Lin ${ }^{1}$, Xinli Song², Guoyang $\mathrm{Wu}^{2}$ and $\mathrm{Zhida} \mathrm{Su}^{2}$ \\ 1 School of Nuclear Science and Engineering, Shanghai Jiao Tong University, Shanghai 200240, China \\ 2 China Electric Power Research Institute, Beijing 100192, China
}

\begin{abstract}
The simulation technology of the nuclear power plant are gradually applying to the nuclear power industry. However, most of the research on nuclear power plant simulation system only focus on pure computerized simulation at present, and it is difficult to fully display the characteristics of the simulating objects. In order to simulate the response characteristics of control system more really, a hardware-in-loop simulation platform of main control systems in the nuclear power plant has been developed in this paper. This simulation platform consists of thermalhydraulic model, control and protection system model, physical DCS system and real-time interactive database. A physical industrial DCS system has been coupled to this platform to simulate the main control systems in the NPP, which makes the simulation result much closer to the actual control systems. The devoloped simulation platform has been validated by some steady and transient cases in this paper. This hardware-in-loop simulation platform can be used in the simulation and optimal design of NPP control systems. Furthermore, it can be used in the failure mode and effect analysis of the instrumentation and control systems in the nuclear power plant.
\end{abstract}

\section{Introduction}

The nuclear power plant systems have the characteristics of high construction cost, the professionalism of operation and the risk of improper handling of the incident. Therefore, simulation tests shall be done before a nuclear power plant system or a new reactor is put into operation, including the power up and down in normal operation, the response of the parameters during transient operation, and the action of controlling and protection and so on. However, most of the research on nuclear power plant simulation system only focus on pure computerized simulation at present, which means the whole models are realized by computer codes. Pure computerized simulation is difficult to simulate the characteristics of the simulation objects perfectly, especially the simulation of digital instrumentation and control systems. The digital instrument control system is widely used in the new nuclear power plants or the upgrade. This new technology has produced many benefits as well as new problems in the safety and reliability of nuclear power plants[1].In order to simulate the real characteristics of control systems, it is necessary to develop a set of the hardware-in-loop simulation platform of NPP control system.

A pure computerized simulator of NPP has been developed in our laboratory, and this simulator has been used in the verification and validation of the digital I\&C systems and reactor protection system in the CPR1000type nuclear power plants of China, such as Ling Ao,
Hong Yan He, Yang Jiang nuclear power plants[2-5]. Based on this NPP simulator, a physical DCS system has been coupled to the NPP simulator to form a hardwarein-loop simulation platform in this paper. By the realtime database and synchronization control, the presented simulation platform can reach real-time operation. After the design of the hardware-in-loop simulation platform, corresponding verification works have also been carried out.

\section{Framework of hardware-in-loop simulation platform}

The presented hardware-in-the-loop simulation platform consists of a physical DCS system, modules of NPP simulator and real-time interactive database. The general framework is shown in Figure 1.

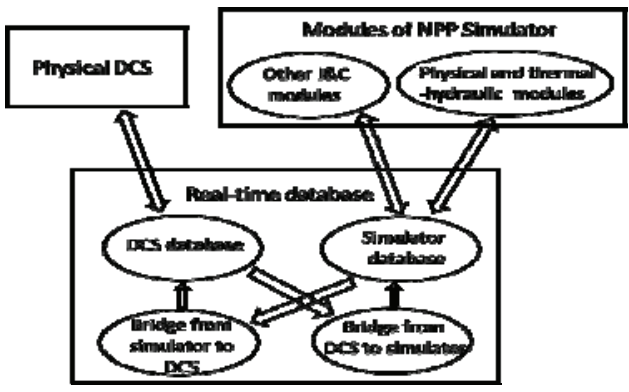

Figure 1. General framework of hardware-in -loop simulation platform

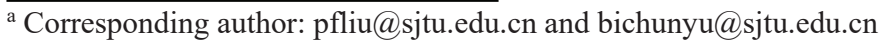


Because of the difference between the two databases of DCS and simulator, we develop two bridges to achieve data conversion and delivery. Due to the limitations of the simulation scale and $\mathrm{I} / \mathrm{O}$ numbers of the physical DCS system, the DCS is only used to develop main control systems in the nuclear power plant. Other auxiliary control systems are simulated in the NPP simulator by Matlab/Simulink tools.

\subsection{Physical and thermal-hydraulic modules}

Physical and thermal-hydraulic modules in the NPP simulator are developed by RELAP5. RELAP5 is a onedimensional, unbalanced and two-phase thermal hydraulic system program developed by Idaho National Engineering Laboratory. It is widely used in nuclear industry for numerical simulation and safety analysis. Figure 2 shows thermal-hydraulic nodalization scheme of the primary loop of a CPR1000-type nuclear power plant in this simulation platform. It includes a reactor vessel, a pressurizer, three loops (which each has a steam generator and circulating pump) and other auxiliary systems. The secondary loop and other auxiliary systems are also included in the simulation platform.

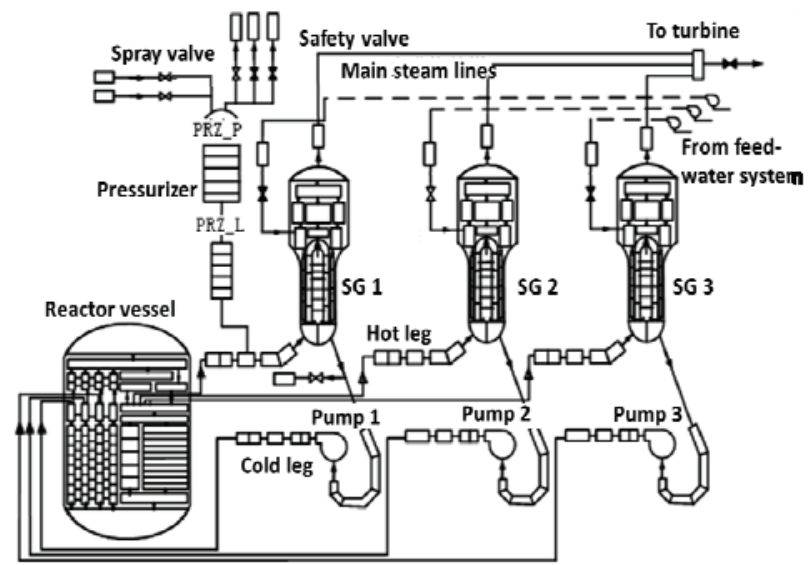

Figure 2. The thermal-hydraulic node diagram of primary loop

\subsection{Physical DCS and control systems}

A distributed control system named XDC800 of Xinhua Company is used in the hardware-in-loop simulation platform, which has been shown in Figure 3. It is made up of three DCS cabinets and a computer server. The DCS cabinets have configured $\mathrm{I} / \mathrm{O}$ modules and controllers, which is to realize the signal acquisition, processing and logic control. The computer server has configured DCS software, which is to realize channel configuration, logical configuration and human-machine interface. The DCS server has also be used as the server of NPP simulator. XDC800 uses redundant communications and controller strategies to improve reliability. Ethernet is used as communication networks in the platform. The physical DCS in the simulation platform can achieve analog-to-digital signal conversion from the DCS to simulator, and digital-to-analog conversion from the simulator to DCS, and real-time transmission and control of data.

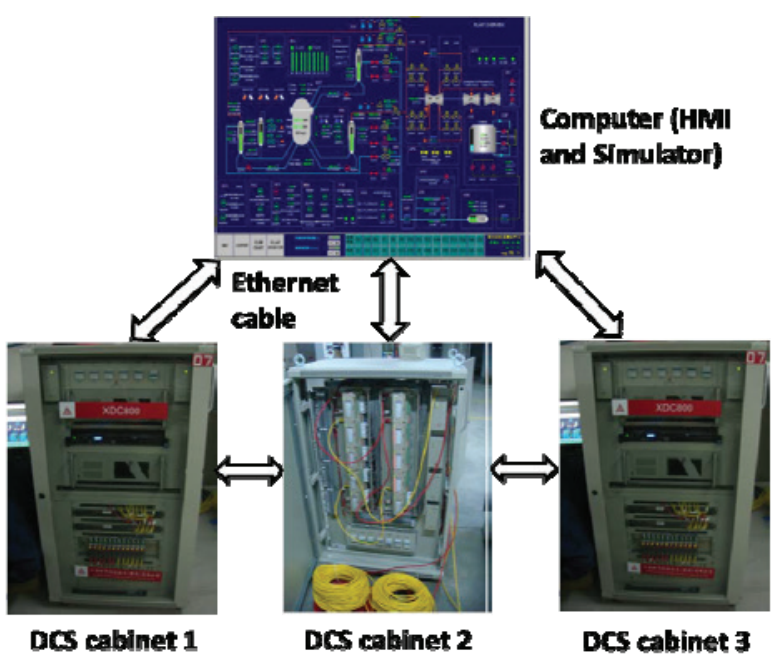

Figure 3. Physical DCS system

This physical DCS has more than $400 \mathrm{I} / \mathrm{O}$ points and it can meet the simulating requirement of main control systems in the nuclear power plant. The developed control systems include main control system of NPP, such as reactor power control system, pressurizer pressure and water level control system, and water level control system of the steam generator, and load control system of the turbine, steam dump control system, and so on. By taking pressurizer pressure control system as an example, we will introduce the modelling progress of the control system in the DCS.

The main function of the pressurizer pressure control system is to maintain the pressure at its nominal value of $15.5 \mathrm{MPa}$ (absolute pressure) in the primary loop. The executive structure is spray valves at top of pressurizer and heaters at the bottom. The control system schematic is given as Figure 4. By taking pressure differential between the measured value and reference value as input signal, a PID controller will regulate the opening of two spray valves and heating power. There are three low-level alarm and two high-level alarm to protect the pressurizer. In general, if the pressure is higher than reference value, the controller will increase the opening of spray valve and decrease the heating power. This ensures that the normal transient will not cause an emergency shutdown of the reactor, nor will the safety valve open.

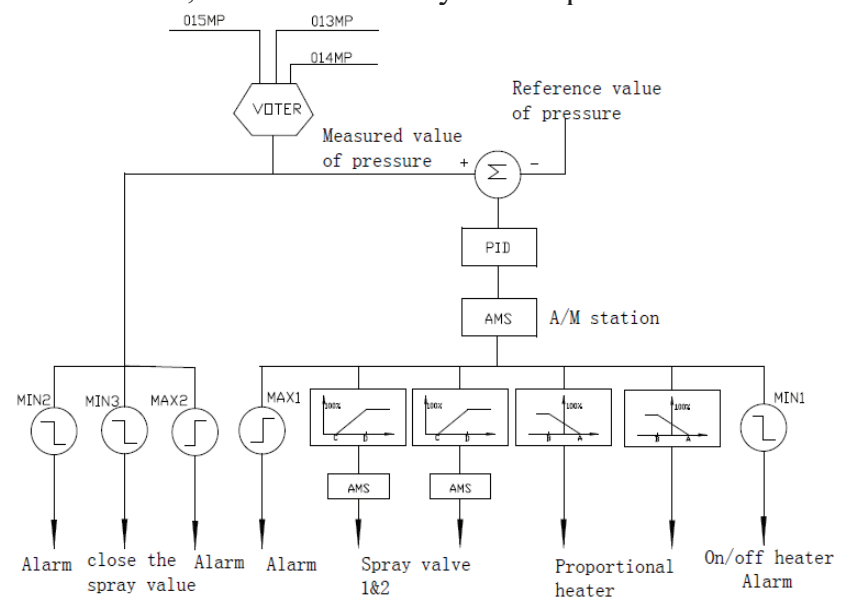

Figure 4. Pressurizer pressure control system schematic 
According to the pressure control schematic, the control logic can be realized as shown in Figure 5 by DCS configuration tools.

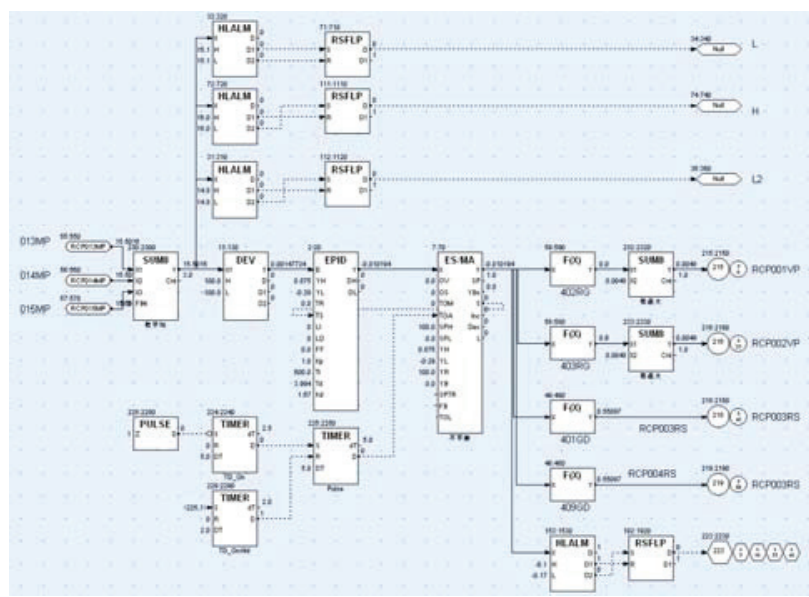

Figure 5. Logic configuration of pressurizer pressure control system in DCS

\subsection{Real-time database and synchronization}

Real-time operating and synchronization control are key characteristics in the hardware-in-loop simulation. The DCS system and database can achieve operation in realtime very easily. Most of the important is how to achieve the real-time operation of the NPP simulator. As we know, the simulation time is up to the scale and algorithm of the simulation platform. Because the physical and thermal-hydraulic modules of nuclear power plant are very large and complex, the RELAP simulation would cause the run-time failure of the simulation in a real-time mode. Our solution is to divide the whole RELAP5 model into parts and use two or more RELAP5 modules to perform the same simulation. This parallel calculating mode can increase the calculating speed to achieve operation in real-time of the simulator. The method of time synchronization control has been described in detail at [6]. As shown in the Figure 6, we set a synchronization time $\Delta \mathrm{t}_{\text {syn }}$ before simulation, when the simulation start, the fast modules (like DCS processing) will wait until the synchronization time finishes at a step. Therefore, the developed hardware-in-loop simulation platform can operate in real-time as long as the calculating time step of RELAP modules is small enough. The calculating time step of RELAP modules can be set to $100 \mathrm{~ms}$ or less in this simulation platform.

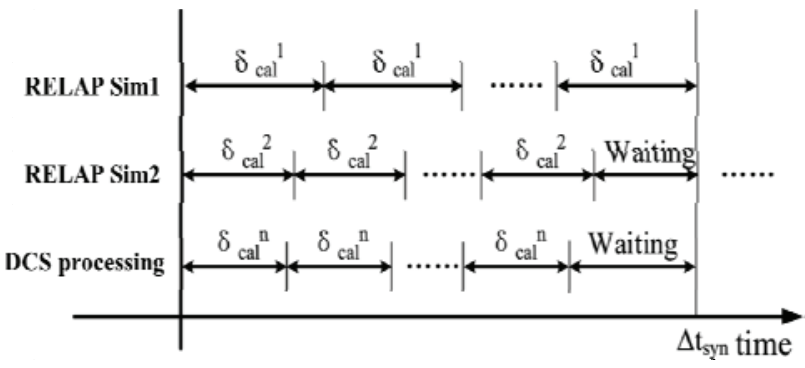

Figure 6. Time synchronization control of simulation platform

\section{Validation of the simulation platform}

After the hardware-in-loop simulation platform has been developed, much validation works should be done. As we mentioned in the introduction, we have developed a pure computerized NPP simulator from 2005, and it has been used to V\&V of digital control and protection systems in Chinese CPR1000-type nuclear power plants. Here we use pure computerized NPP simulator as the reference to validate the hardware-in-loop simulation platform.

The validation work include steady state at different power level and all kinds of normal transients in the nuclear power plant. Here we just give the two examples as follows.

\subsection{Steady state at full power}

The simulation platform should achieve steady states at the different power stage firstly. Figure 7 gives history of main parameters of the primary loop at $100 \% \mathrm{FP}$ steady state. The simulation results show that the designed hardware-in-loop simulation platform can achieve and keep the steady state at $100 \% \mathrm{FP}$. The main parameters are very close to nominal value and the steady state error is less than $0.2 \%$.
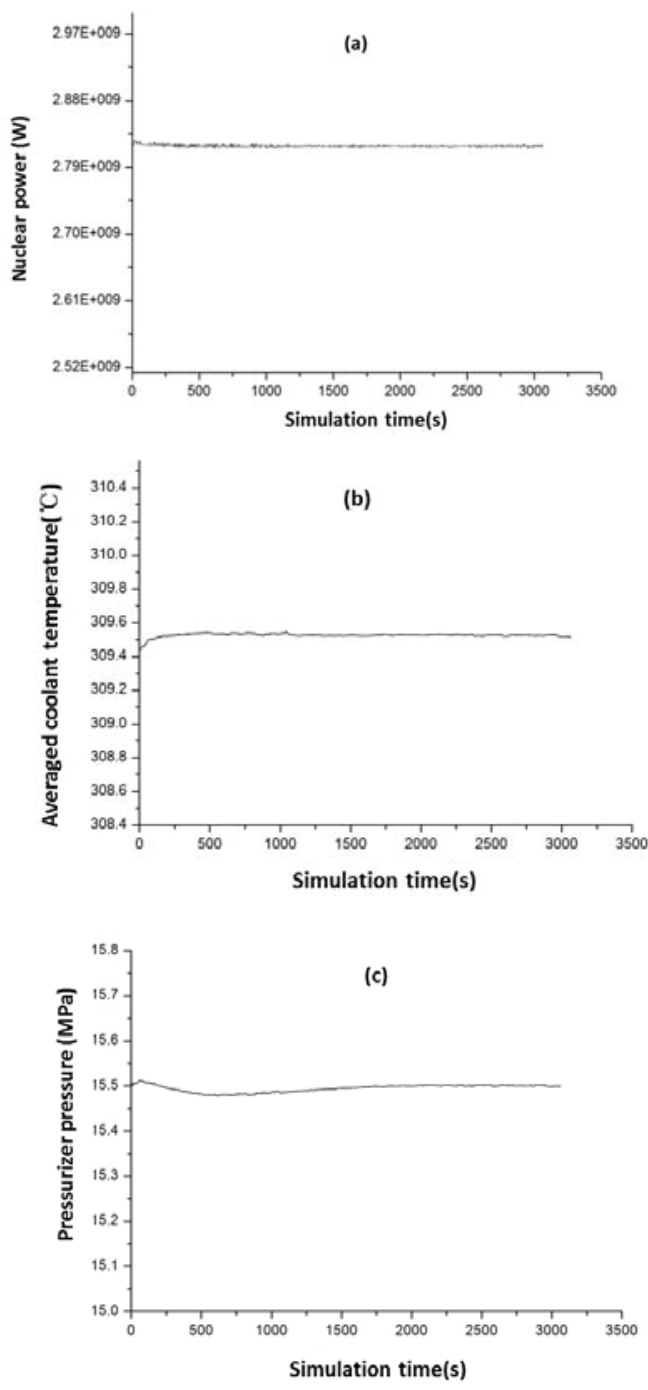


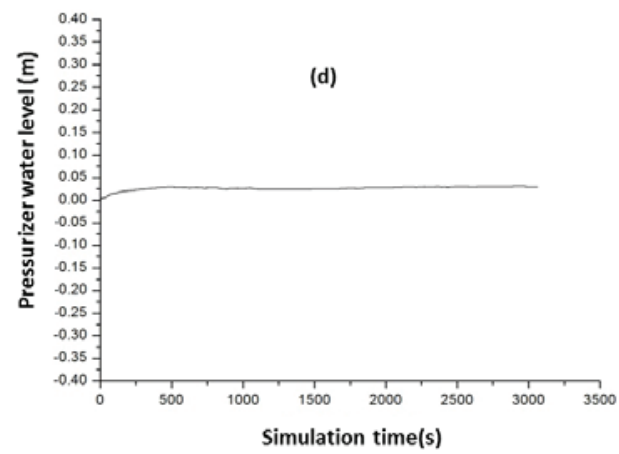

Figure 7. History of main primary loop parameters at $100 \% \mathrm{FP}$ steady state

\subsection{Load ramp transient from $100 \% \mathrm{FP}$ to $15 \% \mathrm{FP}$}

To verify the load tracking ability and the adjustment ability of the hardware-in-the-loop simulation platform, some step load and linear load change tests have been carried out. A load ramp transient from $100 \% \mathrm{FP}$ to $15 \% \mathrm{FP}$ at $5 \% \mathrm{FP} / \mathrm{min}$ rate is given as an example.

Figure 8 shows the history of main parameters during the load ramp transient. From the figure, we can see that the turbine load changes firstly, and then nuclear power tracks the load change by regulating the reactor power control system. The steady value of nuclear power will be bigger than turbine load, this is due to activate of the final power setting value. After transient fluctuations, coolant averaged temperature, pressure and water level of the pressurizer are eventually stabilized at the nominal values corresponding to the $15 \% \mathrm{FP}$. The results indicate that all the main control systems have play roles. The comparison results between the hardware-in-loop simulation platform and pure computerized simulator show that the overall trend of the transient and the final steady value are consistent, but there are some differences. For example, the overshoot of hardware-inloop platform is a little bigger than that of pure computerized simulator. The simulation result of the hardware-in-loop also shows some characteristic of the digital system, such as fluctuations caused by signal interference and resolution.

\section{Conclusions}

In this paper, the industrial DCS configuration and cabinet are applied to the NPP control systems simulation platform, and this can improve the simulation results by considering the response characteristic of the digital I\&C systems in the nuclear power plant. The presented validation shows that this hardware-in-loop simulation platform can simulate all kinds of steady state and transient of plants. It means that this simulation platform can be used to the simulation and optimal design of NPP digital control systems. Furthermore, due to a physical DCS including in this platform, it can also be applied to reliability analysis of digital systems in the future.
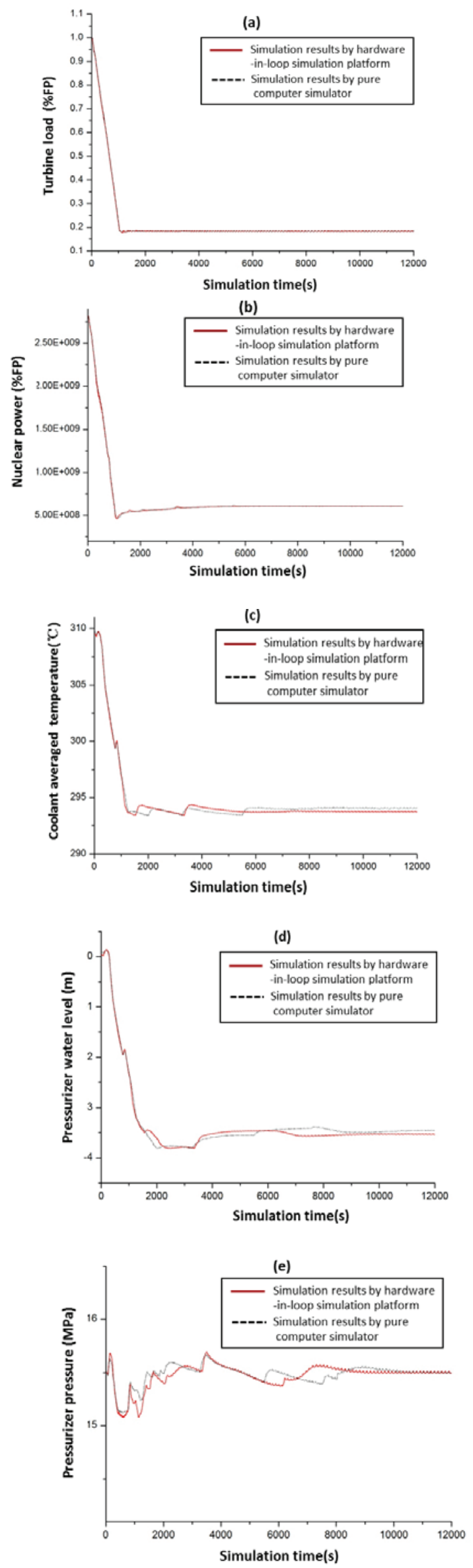

Figure 8. Historyof main parameters at load ramp transient 


\section{Acknowledgment}

Authors are grateful to State Grid Corporation Science and Technology Project of China (No: XT71-15-060) and China Electric Power Research Institute Innovation Fund Project (No: XT83-15-002) for providing the financial support for this study.

\section{References}

1. National Research Council, Digital Instrumentation and Control Systems in Nuclear Power Plants: Safety and Reliability Issue.(National Academy Press, 1997)

2. Liu P., Lin M., Hou D., et al., Research on engineering eimulator for function validating of DCS in nuclear power plant. Nucl. Power. Eng.,30(5s),4851(2009)

3. Lin M., Su Y., Hu R., et al., Development of a thermal-hydraulic system code for simulators based on RELAP5 code. Nucl. Eng. Des.,235 (6), 675686(2005)

4. Lin M., Hou D., Liu P., et al., Main control system verification and validation of NPP digital I\&C system based on engineering simulator. Nucl. Eng. Des. ,240, 1887-1896(2010)

5. Hou D., Lin M., Xu Z., et al., Development and application of an extensible engineering simulator for NPP DCS closed-loop test. Ann. Nuc. Energy, 38,49-55 (2011)

6. Li Y., Lin M., Yang Y., Coupling methods for parallel running RELAPSim codes in nuclear power plant simulation. Nucl. Eng. Des. ,297, 1-14(2016) 